\title{
FAult Isolation in TEChNiCAL SySTEMS BY DATA-DRIVEN METHOD
}

\author{
Alexey Zhirabok ${ }^{\mathrm{a}, \mathrm{b}}$, Filaretov Vladimir ${ }^{\mathrm{a}}$, Sergey Pavlov ${ }^{\mathrm{a}}$ \\ ${ }^{a}$ Far Eastern Federal University, Sukhanova street, 8, Vladivostok, 690990, Russia \\ ${ }^{b}$ Institute of Applied Mathematics, Radio street, 7, Vladivostok, 690041, Russia
}

\begin{abstract}
The paper is devoted to the problem of fault isolation in technical systems described by linear dynamic models. The data-driven method providing a possibility to consider the systems with unknown parameters is used. To isolate faults, the special parallel decomposition of the initial system is suggested. This decomposition contains several subsystems such that the i-th subsystem is free from the $i$-th fault. This property allows to consider the faults independently of one another and to develop the fault isolation procedure. To construct such decomposition, special algorithm is suggested. To apply the data-driven method, each subsystem should be transformed into well-known identification canonical form. The theoretical results are illustrated by the example.
\end{abstract}

Keywords: linear systems; diagnosis; fault isolation, data-driven method
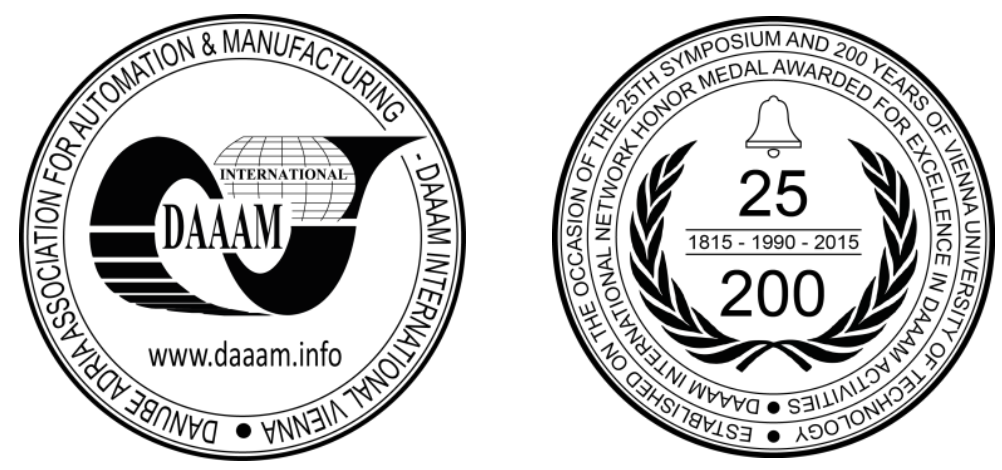

This Publication has to be referred as: Zhirabok, A[lexey]; Filaretov, V[ladimir] \& Pavlov, S[ergey] (2016). Fault Isolation in Technical Systems by Data-Driven Method, Proceedings of the 26th DAAAM International Symposium, pp.0118-0124, B. Katalinic (Ed.), Published by DAAAM International, ISBN 978-3-902734-07-5, ISSN 1726-9679, Vienna, Austria DOI: $10.2507 / 26$ th.daaam.proceedings.017 


\section{Introduction}

The problem of fault diagnosis in technical systems was extensively investigated for the past 25 years; see, e.g., the papers $[6,7,8,13,14,16,18]$, the books [2, 3, 9-12]. Many problems have been studied and solved: different methods of residual generation and relationships among them, robustness and adaptive threshold test, $H_{\infty}$ approach, fuzzy logic. Many types of systems have been considered: linear, bilinear, linearly-analytical, descriptor, different classes of nonlinear systems. Many practical examples have been considered; see, for example, books devoted to industrial and mechatronic systems $[3,11,12]$.

There exists a promising method of fault diagnosis known as "model-free" or "data-driven" method developed for fault detection in technical systems described by linear models mainly $[1,4,5,15,17]$. The feature of this method is that parameters of the system under consideration may be unknown. Therefore, this method may be named as the nonparametric one as well.

The peculiarity of the method developed in $[1,4,15]$ lies in the fact that it allows to detect faults only but not isolate. However, many practical applications need in more precise information about the faults, i.e. they need in fault isolation. Timely and exact fault isolation allows to reduce the working expenses. The purpose of the present paper is to develop the procedure based on the data-driven method which allows to isolate the faults.

The method suggested in $[1,4,15]$ assumes that diagnostic procedure uses the matrix $V$ built on the basis of the measurements $(u(t), y(t)), t=0,1, \ldots, T$, for some temporal interval $T$, where $u(t)$ and $y(t)$ are vectors of control and output, respectively. Decision making is based on the kernel of the matrix $V$ using to generate the residual.

\section{Preliminaries}

Consider the discrete-time linear control system described by the model

$$
x(t+1)=F x(t)+G u(t)+\sum_{i=1}^{s} D_{i} \gamma(t), y(t)=H x(t)
$$

where $x \in X \subseteq R^{n}, u \in U \subseteq R^{m}$, and $y \in Y \subseteq R^{l}$ are vectors of state, control and output; $F, G, H$, and $D_{i}$ are constant matrices, one assumes that the matrix $F$ is nonsingular; $\gamma=\left(\gamma_{1}, \gamma_{2}, \ldots, \gamma_{s}\right)^{\mathrm{T}}$ is the vector corresponding to the faults: for the healthy system $\gamma_{i}=0$, if the $i$-th fault occurs, $\gamma_{i}(t)$ becomes an unknown function of time, $i=1,2, \ldots, s$. Notice that non-singularity of the matrix $F$ is a limitation on the class of systems under diagnosis.

The main idea of fault isolation suggested in the paper is based on the decomposition of the initial systems shown in Figure 1 . Here $\Sigma_{1}, \Sigma_{2}, \ldots, \Sigma_{p}$ are some subsystems, $y^{(1)}, y^{(2)}, \ldots, y^{(p)}$ are some linear functions of the output vector $y$ :

$$
y^{(1)}=R^{(1)} y, y^{(2)}=R^{(2)} y, y^{(p)}=R^{(p)} y
$$

Here $p \leq l, p \leq s ; R^{(1)}, R^{(2)}, \ldots, R^{(p)}$ are some matrices to be determined.

To solve the problem under consideration, one assumes that elements of the vector $\gamma=\left(\gamma_{1}, \gamma_{2}, \ldots, \gamma_{s}\right)^{\mathrm{T}}$ are distributed among $p+1$ sets $\Gamma=\left\{\Gamma_{0}, \Gamma_{1}, \Gamma_{2}, \ldots, \Gamma_{p}\right\}$ so that the subsystem $\Sigma_{1}$ does not contain the elements from the set $\Gamma_{1}$, the subsystem $\Sigma_{2}$ from the set $\Gamma_{2}$ and so on; the elements from $\Gamma_{0}$ are contained in all subsystems.

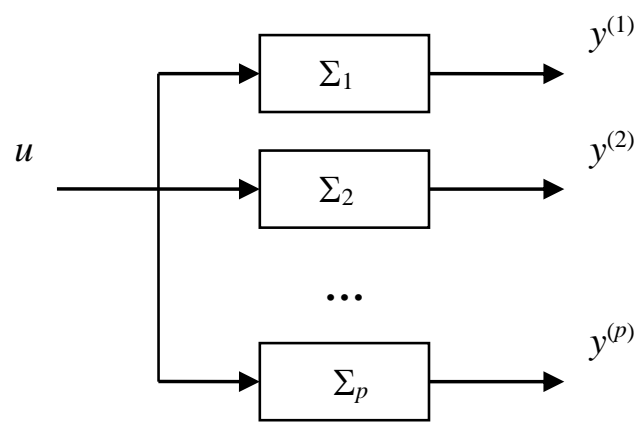

Fig. 1. Decomposition of the initial system 
To isolate the faults, the diagnostic procedure considered in $[1,4,15]$ is applied to the subsystems $\Sigma_{1}, \Sigma_{2}, \ldots, \Sigma_{p}$ by finding the matrices $V^{(1)}, V^{(2)}, \ldots, V^{(p)}$ built on the basis of the measurements

$\left(u(t), y^{(1)}(t)\right), t=0,1, \ldots, T_{1},\left(u(t), y^{(2)}(t)\right), t=0,1, \ldots, T_{2},\left(u(t), y^{(p)}(t)\right), t=0,1, \ldots, T_{p}$

respectively. The residuals $r_{1}, r_{2}, \ldots, r_{p}$ are generated on a basis of the data-driven method, and decision making is based on the binary matrix $S$ of fault syndromes

$S=\left(\begin{array}{ccccc}1 & 0 & 1 & \cdots & 1 \\ 1 & 1 & 0 & \cdots & 1 \\ \vdots & \vdots & \vdots & \ddots & \vdots \\ 1 & 1 & 1 & \cdots & 0\end{array}\right)$

where rows correspond to the residuals $r_{1}, r_{2}, \ldots, r_{p}$, columns correspond to the sets $\Gamma_{0}, \Gamma_{1}, \Gamma_{2}, \ldots, \Gamma_{p} ;$ the symbols 0 and 1 correspond to $r_{i}=0$ and $r_{i} \neq 0$, respectively. Clearly, the fault isolation procedure can be achieved with an accuracy of the sets $\Gamma_{0}, \Gamma_{1}, \ldots, \Gamma_{p}$.

\section{Subsystem $\Sigma_{i}$ construction}

Assume that the subsystem $\Sigma_{i}$ is described by the equations similar to (1):

$x^{(i)}(t+1)=F^{(i)} x^{(i)}(t)+G^{(i)} u(t)+\sum_{j \neq i}^{s} D^{(j)} \gamma, y^{(i)}(t)=H^{(i)} x^{(i)}(t)$

where $F^{(i)}, G^{(i)}, H^{(i)}$, and $D^{(j)}$ are some matrices to be determined. It is known that the state $x^{(i)}(t)$ is a linear combination of the system (1) state $x(t)$ according to

$x^{(i)}(t)=\Phi^{(i)} x(t)$

for some matrix $\Phi^{(i)}$ in the unfaulty case after the response to unlike conditions has died out. Rewriting (3) for $t+1$ and using (1) and (2), one obtains

$\Phi^{(i)} F=F^{(i)} \Phi^{(i)}, \Phi^{(i)} G=G^{(i)}, \Phi^{(i)} D_{i}=0, H^{(i)} \Phi^{(i)}=R^{(i)} H$

To construct the subsystem $\Sigma_{i}$, introduce the matrix $D^{i}$ of maximal rank such that $D^{i} D_{i}=0$. In this case, the condition $\Phi^{(i)} D_{i}=0$ implies the equality $\Phi^{(i)}=M D^{i}=0$ for some matrix $M$.

To find the matrix $\Phi^{(i)}$ satisfying the condition (4), construct a sequence of matrices $P^{0}:=D^{i}, P^{1}, P^{2}, \ldots$ as follows. The matrix $P^{1}$ is specified by $P^{1}:=N_{0} P^{0}$, where the matrix $N_{0}$ is a solution of the algebraic equation

$N\left(\begin{array}{c}P^{0} \\ P^{0} F^{-1}\end{array}\right)=0$

represented in the form $N=\left(\begin{array}{ll}N_{0} & N_{0}^{\prime}\end{array}\right)$. By analogy, set $P^{i+1}:=N_{i} P^{i}$, where $N_{i}$ is a solution of the algebraic equation

$N\left(\begin{array}{c}P^{i} \\ P^{i} F^{-1}\end{array}\right)=0, i=0,1, \ldots$

represented in the form $N=\left(\begin{array}{ll}N_{i} & N_{i}^{\prime}\end{array}\right)$. The final step $j$ is specified by the condition

$\operatorname{rank}\left(P^{j+1}\right)=\operatorname{rank}\left(P^{j}\right)$

The last condition means that the matrix $N_{j}$ is nonsingular.

Equation (6) with $i=j$ yields the expression 
$N\left(\begin{array}{c}P^{i} \\ P^{i} F^{-1}\end{array}\right)=N_{j} P^{j}+N_{j}^{\prime} P^{j} F^{-1}=0$

or $N_{j} P^{j} F+N_{j}^{\prime} P^{j}=0$

Theorem. The matrix $\Phi^{(i)}:=P^{j}=N_{j} \ldots N_{0} D^{i}$ satisfies the first condition in (4) for some matrix $F^{(i)}$. Besides, it is of maximal rank, and its rows are linear combinations of rows of the matrix $D^{i}$, i.e. $\Phi^{(i)}=M D^{i}$ for some matrix $M$.

Proof. The last part follows immediately from the definition of matrix $\Phi^{(i)}$. Denoting $F^{(i)}=-N_{j}^{-1} N_{j}^{\prime}$, one obtains from (7) the first equality in (4) that proves the first part. To prove the rest, consider some matrix $K$ satisfying the conditions $K F=F_{*}^{\prime} K$ and $K=Q D^{i}$ for some matrices $F_{*}^{\prime}$ and $Q$. Replace the matrix $K$ with $Q D^{i}=Q P^{0}$ :

$Q P^{0} F=F_{*}^{\prime} Q P^{0}$

and rewrite the obtained expression in the form

$\left(\begin{array}{ll}Q & -F_{*}^{\prime} Q\end{array}\right)\left(\begin{array}{c}P^{0} \\ P^{0} F^{-1}\end{array}\right)=0$

where known matrices are separated from the unknown ones. Since $N=\left(\begin{array}{ll}N_{0} & N_{0}^{\prime}\end{array}\right)$ is a solution of equation (5), one obtains $\left(Q-F_{*}^{\prime} Q\right)=C_{0}\left(N_{0} \quad N_{0}^{\prime}\right)$ for some matrix $C_{0}$, in particular, $Q=C_{0} N_{0}$. Therefore, $K=C_{0} N_{0} P^{0}=C_{0} P^{1}$. It can be shown by analogy that $K=C_{1} P^{2}, \ldots, K=C_{j-1} P^{j}=C_{j-1} \Phi^{(i)}$ for some matrices $C_{1}, \ldots, C_{j-1}$. This means that the matrix $\Phi^{(i)}$ is of maximal rank. The theorem has been proved.

To construct the subsystem $\Sigma_{i}$, set $G^{(i)}:=\Phi^{(i)} G$ and solve the algebraic equation

$B\left(\begin{array}{c}H \\ \Phi^{(i)}\end{array}\right)=0$

If this equation has a solution, rewrite the matrix $B$ in the form $B=\left(\begin{array}{ll}B_{0} & B_{0}^{\prime}\end{array}\right)$ and set $R^{(i)}:=B_{0}$ and $H^{(i)}:=-B_{0}^{\prime}$, since in this case (8) implies the last equation in (4). Notice that since the matrix $\Phi^{(i)}$ is of maximal rank, equation (8) has the best possibility to be solved. Thus, the subsystem $\Sigma_{i}$ has been built; the rest subsystems are constructed analogously. If $\Phi^{(i)}=P^{j}=N_{j} \ldots N_{0} D^{i}=0$ or equation (8) has not a solution, the subsystem without the element $\gamma_{i}$ does not exist, in this case this element is included in the set $\Gamma_{0}$.

The constructed subsystems $\Sigma_{1}, \Sigma_{2}, \ldots, \Sigma_{p}$ are used to obtain the parity relations by known methods [1, 4, 15]; the $i$-th subsystem $\Sigma_{i}$ allows to generate the residual $r_{i}, i=1,2, \ldots, p$. The problem of fault isolation can be solved based on the residuals $r_{1}, r_{2}, \ldots, r_{p}$ and the matrix $S$ of fault syndromes.

\section{Illustrative example}

For simplicity, we introduce the following notation: $x^{+}:=x(t+1), x:=x(t), u:=u(t), y:=y(t)$. It is assumed that the system is described by the equations

$$
\begin{aligned}
& x_{1}^{+}=x_{2}+u_{1}+u_{2}+x_{5}+\gamma_{1}+\gamma_{4} \\
& x_{2}^{+}=x_{3}+x_{5}+\gamma_{2} \\
& x_{3}^{+}=-2 x_{4}+x_{1}-u_{1}+u_{2}-x_{5}-\gamma_{3} \\
& x_{4}^{+}=u_{2}+x_{5}+\gamma_{4} \\
& x_{5}^{+}=x_{4}+u_{1}+x_{5}+\gamma_{3} \\
& y_{1}=x_{1}-x_{4} \\
& y_{2}=x_{4}
\end{aligned}
$$


Matrices describing the system are as follows:

$$
\begin{aligned}
F & =\left(\begin{array}{lllll}
0 & 1 & 0 & 0 & 1 \\
0 & 0 & 1 & 0 & 1 \\
1 & 0 & 0 & -2 & -1 \\
0 & 0 & 0 & 0 & 1 \\
0 & 0 & 0 & 1 & 1
\end{array}\right), G=\left(\begin{array}{cc}
1 & 1 \\
0 & 0 \\
-1 & 1 \\
0 & 1 \\
1 & 0
\end{array}\right), H=\left(\begin{array}{ccccc}
1 & 0 & 0 & -1 & 0 \\
0 & 0 & 0 & 1 & 0
\end{array}\right) \\
D_{1} & =\left(\begin{array}{llll}
1 & 0 & 0 & 0 \\
0 & 0 & 0 & 0 \\
0 & 0 & 0 & 0 \\
0 & 0 & 0 & 0 \\
0 & 0 & 0 & 0
\end{array}\right), D_{2}=\left(\begin{array}{llll}
0 & 0 & 0 & 0 \\
0 & 1 & 0 & 0 \\
0 & 0 & 0 & 0 \\
0 & 0 & 0 & 0 \\
0 & 0 & 0 & 0
\end{array}\right), D_{3}=\left(\begin{array}{cccc}
0 & 0 & 0 & 0 \\
0 & 0 & 0 & 0 \\
0 & 0 & -1 & 0 \\
0 & 0 & 0 & 0 \\
0 & 0 & 1 & 0
\end{array}\right), D_{4}=\left(\begin{array}{llll}
0 & 0 & 0 & 1 \\
0 & 0 & 0 & 0 \\
0 & 0 & 0 & 0 \\
0 & 0 & 0 & 1 \\
0 & 0 & 0 & 0
\end{array}\right)
\end{aligned}
$$

Consider the first fault; it can be shown that $D^{1}=\left(\begin{array}{ccccc}0 & 1 & 0 & 0 & 0 \\ 0 & 0 & 1 & 0 & 0 \\ 0 & 0 & 0 & 1 & 0 \\ 0 & 0 & 0 & 0 & 1\end{array}\right)$. According to the theorem, one obtains the matrix $\Phi^{(1)}=\left(\begin{array}{lllll}0 & 0 & 0 & 1 & 0 \\ 0 & 0 & 0 & 0 & 1\end{array}\right)$. Equation (8) yields $R^{(1)}=\left(\begin{array}{ll}0 & 1\end{array}\right)$, therefore $y^{(1)}=y_{2}=x_{2}^{(1)}$. To describe the subsystem $\Sigma_{1}$, set

$$
x^{(1)}=\left(x_{1}^{(1)} \quad x_{2}^{(1)}\right)^{\mathrm{T}}:=\Phi^{(1)} x=\left(\begin{array}{ll}
x_{4} & x_{5}
\end{array}\right)^{\mathrm{T}}
$$

Then $x^{(1)+}=\left(\begin{array}{ll}x_{4}^{+} & x_{5}^{+}\end{array}\right)^{\mathrm{T}}=\left(\begin{array}{ll}u_{2}+x_{5}+\gamma_{4} & x_{4}+u_{1}+x_{5}+\gamma_{3}\end{array}\right)^{\mathrm{T}} ;$ as a result,

$$
\begin{aligned}
& x_{1}^{(1)+}=u_{2}+x_{2}^{(1)}+\gamma_{4} \\
& x_{2}^{(1)+}=x_{1}^{(1)}+x_{2}^{(1)}+u_{1}+\gamma_{3}
\end{aligned}
$$

Analogously, one may find $D^{2}=\left(\begin{array}{ccccc}1 & 0 & 0 & 0 & 0 \\ 0 & 0 & 1 & 0 & 0 \\ 0 & 0 & 0 & 1 & 0 \\ 0 & 0 & 0 & 0 & 1\end{array}\right)$ and $\Phi^{(2)}=\Phi^{(1)}=\left(\begin{array}{ccccc}0 & 0 & 0 & 1 & 0 \\ 0 & 0 & 0 & 0 & 1\end{array}\right)$, i.e. the second subsystem coincides with the first one. Since the description of the subsystem does not contain the elements $\gamma_{1}$ and $\gamma_{2}$, then $\Gamma_{1}=\left\{\gamma_{1}, \gamma_{2}\right\}$.

Considering by analogy the elements $\gamma_{3}$ and $\gamma_{4}$, one obtains $\Phi^{(3)}=\Phi^{(4)}=\left(\begin{array}{ccccc}1 & 0 & 0 & -1 & 0 \\ 0 & 1 & 0 & 0 & 0 \\ 0 & 0 & 1 & 0 & 1\end{array}\right)$ and sets

$x^{(2)}=\left(\begin{array}{lll}x_{1}^{(2)} & x_{2}^{(2)} & x_{3}^{(2)}\end{array}\right)^{\mathrm{T}}:=\Phi^{(3)} x=\left(\begin{array}{lll}x_{1}-x_{4} & x_{2} & x_{3}+x_{5}\end{array}\right)^{\mathrm{T}}$

As a result, the description of the subsystem $\Sigma_{3}=\Sigma_{4}$ is as follows

$$
\begin{aligned}
& x_{1}^{(2)+}=x_{2}^{(2)}+u_{1}+\gamma_{1} \\
& x_{2}^{(2)+}=x_{3}^{(2)}+\gamma_{2} \\
& x_{3}^{(2)+}=u_{2}+x_{1}^{(2)}
\end{aligned}
$$

Clearly, $\Gamma_{2}=\left\{\gamma_{3}, \gamma_{4}\right\}$ and $\Gamma_{0}=\varnothing$. Equation (8) yields $R^{(2)}=\left(\begin{array}{ll}1 & 0\end{array}\right)$, that gives $y^{(2)}=y_{1}=x_{1}^{(2)}$. Clearly, the matrix of fault syndromes is $S=\left(\begin{array}{ll}0 & 1 \\ 1 & 0\end{array}\right)$.

The subsystems $\Sigma_{1}=\Sigma_{2}$ and $\Sigma_{3}=\Sigma_{4}$ can be used to obtain the parity relations and the residuals $r_{1}$ and $r_{2}$, respectively. Thus, the problem of fault isolation can be solved with an accuracy of the sets $\Gamma_{1}=\left\{\gamma_{1}, \gamma_{2}\right\}$ and $\Gamma_{2}=\left\{\gamma_{3}, \gamma_{4}\right\}$.

To start with, the subsystem (9) should be transformed into the identification canonical form. To this end, set $z_{1}:=x_{2}^{(1)}$ and $z_{2}:=x_{1}^{(1)}$; as a result, the model (9) takes the form 
$z_{1}^{+}=z_{2}+z_{1}+u_{1}+\gamma_{3}=z_{2}+y^{(1)}+u_{1}+\gamma_{3}$

$z_{2}^{+}=u_{2}+z_{1}+\gamma_{4}=u_{2}+y^{(1)}+\gamma_{4}$

since

$y^{(1)}=z_{1}$

Then one makes several temporal shifts in (11) to obtain the expression does not containing the components $z_{1}$ and $z_{2}$ :

$$
\begin{aligned}
y^{(1)}(t+1)= & z_{1}(t+1)=z_{2}(t)+y^{(1)}(t)+u_{1}(t)+\gamma_{3}(t) \\
y^{(1)}(t+2)= & z_{2}(t+1)+y^{(1)}(t+1)+u_{1}(t+1)+\gamma_{3}(t+1)= \\
& u_{2}(t)+y^{(1)}(t)+\gamma_{4}(t)+y^{(1)}(t+1)+u_{1}(t+1)+\gamma_{3}(t+1) .
\end{aligned}
$$

According to $[1,4,15]$, the residual $r_{1}$ can be generated as follows:

$$
\begin{aligned}
r_{1}(t)= & \gamma_{4}(t)+\gamma_{3}(t+1)=y^{(1)}(t+2)-\left(u_{2}(t)+y^{(1)}(t)+y^{(1)}(t+1)+u_{1}(t+1)\right)= \\
& y_{2}(t+2)-\left(u_{2}(t)+y_{2}(t)+y_{2}(t+1)+u_{1}(t+1)\right)
\end{aligned}
$$

Clearly, $r_{1}(t)=0$ if $\gamma_{3}(t+1)=\gamma_{4}(t)=0$, i.e. when the initial system is free from the third and fourth faults. Besides, if $\gamma_{3}(t+1) \neq 0$ or $\gamma_{4}(t) \neq 0$, then $r_{1}(t) \neq 0$ except $\gamma_{3}(t+1)+\gamma_{4}(t)=0$.

Since $y^{(2)}=y_{1}=x_{1}^{(2)}$, the subsystem (10) is of identification canonical form already. Make several temporal shifts in $y^{(2)}=x_{1}^{(2)}$ to obtain the expression does not containing the components of the vector $x^{(2)}$ :

$$
\begin{aligned}
& y^{(2)}(t+1)=x_{1}^{(2)}(t+1)=x_{2}^{(2)}(t)+u_{1}(t)+\gamma_{1}(t) \\
& y^{(2)}(t+2)=x_{2}^{(2)}(t+1)+u_{1}(t+1)+\gamma_{1}(t+1)=x_{3}^{(2)}(t)+\gamma_{2}(t)+u_{1}(t+1)+\gamma_{1}(t+1) \\
& y^{(2)}(t+3)=x_{3}^{(2)}(t+1)+\gamma_{2}(t+1)+u_{1}(t+2)+\gamma_{1}(t+2)=u_{2}(t)+y^{(2)}(t)+\gamma_{2}(t+1)+u_{1}(t+2)+\gamma_{1}(t+2)
\end{aligned}
$$

It can be shown that

$$
r_{2}(t)=\gamma_{1}(t+2)+\gamma_{2}(t+1)=y^{(2)}(t+3)-\left(u_{2}(t)+y^{(2)}(t)+u_{1}(t+2)\right)=y_{1}(t+3)-\left(u_{2}(t)+y_{1}(t)+u_{1}(t+2)\right)
$$

Clearly, the residual $r_{2}$ is sensitive to the first and second faults except $\gamma_{1}(t+2)+\gamma_{2}(t+1)=0$.

\section{Conclusion}

The paper is devoted to the problem of fault isolation in technical systems described by linear models. The data-driven method to solve this problem is used. The feature of this method is that parameters of the system under consideration may be unknown. The suggested solution is based on the special decomposition of the initial system. Then the data-driven method is applied to each subsystem of this decomposition. The solution about the faults is based on the set of residuals generated by these subsystems and the matrix of fault syndromes.

The future plan of researches is developing a new algorithm to construct the decomposition shown in Figure 1 in order to overcome the limitation of non-singularity of the matrix $F$.

\section{Acknowledgements}

The publication is supported by the Russian Scientific Foundation (project 16-19-00046).

\section{References}

[1] A. Bittencourt, K. Saarinen, S. Sander-Tavallaey, A Data-driven method for monitoring systems that operate repetitively - applications to wear monitoring in an industrial robot joint, Proc. 8th IFAC Symp. Safeprocess'2012 (2012) pp. 198-203.

[2] M. Blanke, M. Kinnaert, J. Lunze, M. Staroswiecki, Diagnosis and Fault-Tolerant Control, Springer-Verlag, Berlin Heidelberg, 2006. 
[3] F. Caccavale, L. Villiani (Eds), Fault Diagnosis and Tolerance for Mechatronic Systems, Recent Advances, Springer-Verlag, Berlin Heidelberg, 2002.

[4] S. Ding, Y. Wang, S. Yin,P. Zhang, Y. Yang, E. Ding, Date-driven design of fault-tolerant control systems, Proc. 8th IFAC Symp. Safeprocess'2012 (2012) pp. 1323-1328.

[5] V. Filaretov, A. Zhirabok, D. Tkachev, Non-parametric method for fault diagnosis in electrical circuits, Proc. of 23d Int. DAAAM Symposium. Zadar, Croatia (2012) pp. 5-8.

[6] V. Filaretov, M. Vukobratovic, A. Zhirabok, Parity relation approach to fault diagnosis in manipulation robots, Mechatronics, 13 (2003) pp. 141-152.

[7] J. Gertler, Residual generation in model-based fault diagnosis, Control Theory and Advanced Technology, 9 (1993) pp. 259-285.

[8] X. Lou, A. Willsky, G. Verghese, Optimally robust redundancy relations for failure detection in uncertain systems, Automatica, 22 (1996) 333-344.

[9] R. Patton, Robust model-based fault diagnosis: the state of the art, Proc. IFAC Symp. Safeprocess'1994, Finland, Espoo (1994) pp. 1-24.

[10] R. Patton, P. Frank, R. Clark, Issues of fault diagnosis for dynamic systems, Spring Verlag, London, 2000.

[11] E. Russell, L. Chiang, L. Chiang, Fault Detection and Diagnosis in Industrial Systems, Springer-Verlag, Berlin Heidelberg, 2001.

[12] S. Simani, C. Fantuzzi, R. Patton, Model-based Fault Diagnosis in Dynamic Systems Using Identification, Springer-Verlag, Berlin Heidelberg, 2002.

[13] A. Shumsky, A. Zhirabok, Nonlinear diagnostic filter design: algebraic and geometric points of view, Int. Journal of Appl. Math. and Com. Science, 16 (2006) pp. 115-127.

[14] A. Shumsky, Functional diagnosis of nonlinear time-delay dynamic systems, Autom. and Remote Control, 70 (2009) pp. 172-184.

[15] A. Shumsky, Data driven method for fault detection and isolation in nonlinear uncertain systems, CD-ROM Proc. IFAC Conf. on Control Applications in Marine Systems, 2007.

[16] M. Witczak, J. Korbicz, R. Jrozefowicz, Design of unknown input observers for non-linear stochastic systems and their application to robust fault diagnosi, Control and Cybernetics 42 (2013) pp. 1-30.

[17] A. Zhirabok, S. Pavlov, Data-driven method of fault detection in technical systems, Procedia Engineering, 100 (2015) pp. 242-248.

[18] A. Zhirabok, A. Suvorov, A. Shumsky, Robust diagnosis of discrete systems with delay: logic-dynamical approach, Journal of Computer and Systems Sciences International, 53 (2014) pp. 47-62. 